\title{
Meningial and Skull Base Metastasis of Glioblastoma: Case Report and Review of the Literature
}

ElHadji Cheikh Ndiaye SY ${ }^{1}$, Yannick Canton-Kessely ${ }^{2}$, Moussa Diallo ${ }^{3}$, Romain Appay ${ }^{4}$ and Jean Marc Kaya ${ }^{3}$

${ }^{1}$ Neurosurgery Department, Fann's Hospital, Senegal

${ }^{2}$ Neurosurgery Department, Hospital North Marseille, France

${ }^{3}$ Neurosurgery Department, Hopitaux Civils De Colmar, France

${ }^{4}$ Neurosurgery Department, Hospital Timone Marseille, France

*Corresponding author: El hadji Cheikh Ndiaye SY, Neurosurgery Department, Fann's Hospital, Senegal

Submission: January 21, 2018; Published: February 23, 2018

\begin{abstract}
Glioblastoma is the most aggressive glial tumor of the central nervous system in adults. It's growth has always been described as a local invasion and it's direct extension through the dura mater to the skull base is a rare phenomenon. In this paper, we report the unusual case of a 52-year-old patient with disorder language in whom a left temporal lesion was detected on cerebral magnetic resonance imaging (MRI). He underwent a microsurgical resection and the histological examination of the biopsy sample revealed the diagnosis of glioblastoma. Ten months after surgery and concurrent radiochemotherapy, the patient showed visual field degradation and hypoesthesia of the left hemiface. MRI showed contrast-enhanced in the skull base with an extension to the spheno-ethmoidal recess requiring an endoscopic endonasal biopsy which histological examination revealed recurrence of glioblastoma. A targeted therapy was initiated and MRI showed a clear regression of the process at the skull. This report examines the glioblastoma metastases mechanisms outside the central nervous system and the value of targeted therapy in the prognosis of these lesions.
\end{abstract}

Keywords: Glioblastoma; Metastasis meningial; Skull base

\section{Introduction}

Glioblastoma (GB) is the most common malignant central nervous system wich has invasive growth characteristics [1]. Some cases of glioblastoma metastases are reported in the literature. The improvement of the standard of care prolongs the patient's survival and allows detection of metastases [2]. Different attempts have been made to explain the mechanism of extraneuronal metastasis of glioblastoma. We report the case of a left temporal glioblastoma with meningeal and skull base metastasis arising from the dural invasion shown ten months after the surgical excision and after a concurent radio-chemotherapy.

\section{Case Report}

A 51-year-old, right-handed, smoking patient without history antecedent was admitted to emergencies service for a language disorder. Clinical examination revealed a psychomotor slowdown with drowsiness; a Karnofski Performance Status (KPS) evaluated at 60 ; a confusional syndrome and a global Wernicke aphasia on both the auditory and visual sides. CT brain scan realized in emergency showed a voluminous compressive temporal left process, with a mass effect on the median elements and a beginning of contralateral hydrocephalus.

Brain MRI revealed left temporal heterogeneously enhancing lesion, voluminous with signs of engagement. The radiological profile was highly suggestive of glioblastoma (Figure 1).
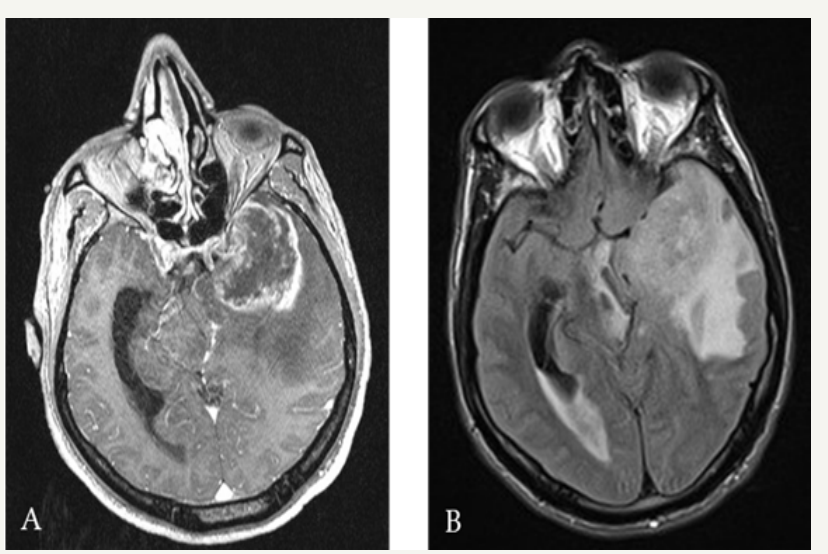

Figure 1: Preoperative contrast-enhanced, axial T1weighted magnetic resonance images: (A) internal temporal and temporo-polar mass measured at $35 \times 47 \mathrm{~mm}$. Heterogeneously enhanced lesion with central necrosis (B) important perilesional oedema with left ventricular enlargement and left temporal engagement and mass effect on the left midbrain and on the chiasma.

The patient underwent surgical resection of the left temporal lesion. Histological examination revealed a largely necrotic tumor proliferation consisting of fusiform cellular elements with a high nucleocytoplasmic ratio of atypical glial-like appearance. There is a vascularization represented by glomerular vessels.

Immunodetections carried out a positivity of the cellular elements with the anti-GFAP antibody. No immunostaining was 
observed either with the anti-Olig2 antibody nor the epithelial markers except for AE1 / AE3 which showed cross-positivity with GFAP. EGFR marked about $60 \%$ of the cells with an intensity at 2. Eighty percent of the nuclei expressed the P53 protein. The proliferation index evaluated with the anti-Ki67 antibody was in the most active territories of about $25 \%$.

There was no immuno-expression with the anti-IDH1 R132H antibody. There was a diffuse positivity in the morphologically "conserved" territories with the anti-ATRX antibody. These histological and immunohistochemical characteristics confirmed the diagnosis of glioblastoma (Figure 2).

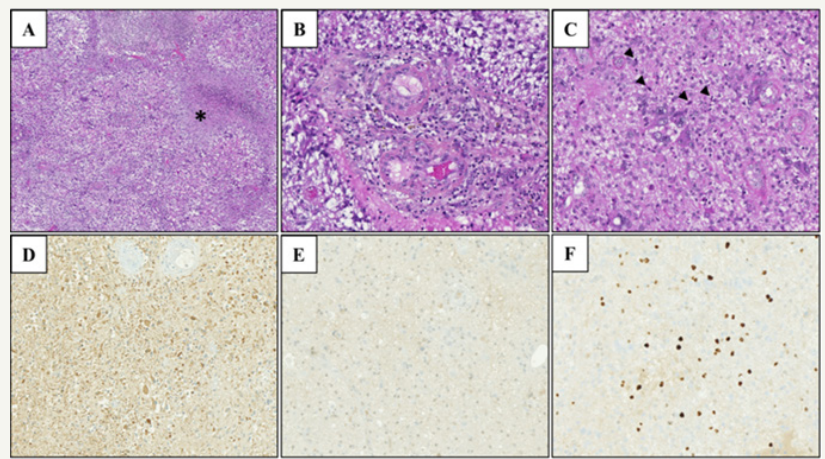

Figure 2:Histopathological examination: A: tumor proliferation with necrosis range $\left({ }^{*}\right)$ B: Presence of microvascular proliferation, C: Pleomorphic proliferation of tumor cells with mitoses (arrows), D: Strong immunoreactivity of neoplastic cells for GFAP, E: negativity of immuno-expression with the anti-IDH1 R132H antibody, F: Presence of proliferation with anti-Ki-67 antibody, Magnification x50 (A), and x200 (B, C, D, E, F).
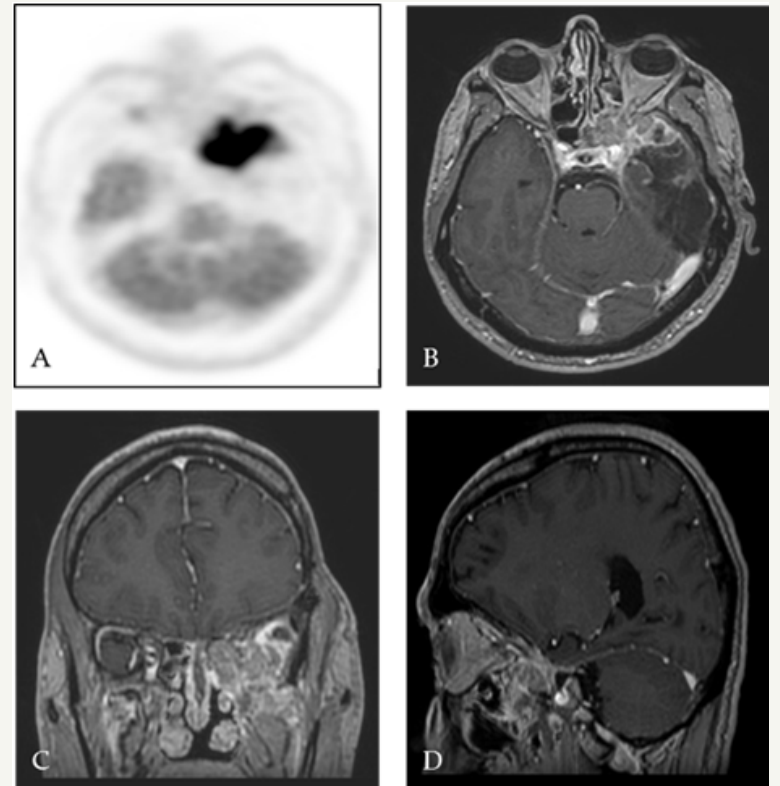

Figure 3: TEP-FDG (A) and contrast-enhanced T1 weighted MRI with gadolinium in axial (B), coronal (C) and sagittal (D) sections ten months after surgery: Presence of an intense hypermetabolic lesion affecting the skull base at the level of the large wing of the sphenoid on the left (ratio of attachment lesion on cortex at 1.7) with contrast-enhancing in the same place.
Given the non-feasibility of radiotherapy due to cognitive status, neoadjuvant chemotherapy has been introduced. Four monthly neoadjuvant cycles of Temozolomide $(200 \mathrm{mg} / \mathrm{m} 2$ or $330 \mathrm{mg} / \mathrm{d}$ ), and a concomitant radio-chemotherapy according to the STUPP protocol, followed by 6 additional monthly temozolomide cures were realized. This treatment had led to a marked improvement in Wernicke's aphasia with recovery of autonomy already before concomitant treatment and a very good radiological response.

Ten months after surgery, it was noted the occurrence of ascending epigastric sensations associated with piloerection which occurred 2 to 3 times a day evocative of partial epileptic seizures of the temporal lobe. There was also a numbness in the territory of the left maxillary nerve (V2) with pains in the left temporozygomatic region. A cerebral PET SCAN FDG of end-of-treatment was performed showing a hypermetabolic focus at the skull base in contact with the left temporal operative cavity and cerebral MRI control showed a contrast in the same place in contact with the left wall of the left sphenoidal sinus also invading the round foramen with extension to the left pterygoid muscles (Figure 3).

Endoscopic endonasal biopsy of the spheno-ethmoidal recess was performed and the histological aspect was consistent with recurrence of glioblastoma. At 14 months postoperative the patient had a persistence of some understanding disorders and hypoesthesia at the level of the left V2. On the other hand, there was a rapid visual degradation with a decrease in visual acuity of the left eye. After a multidisciplinary consultation meeting, in view of the rapid visual degradation and taking into account the unmethylated MGMT status, an oncological treatment by targeted therapy of the Avastin type (10 mg / kg or $600 \mathrm{mg} / \mathrm{d}$ ) with Carboplatin / AUC4 or $630 \mathrm{mg} / \mathrm{d}$ combined with 60 Gy radiotherapy delivered in 30 fractions. Sixteen months after surgery the patient remained clinically stable and cerebral MRI control showed a clear regression of the lesion at the base of the skull (Figure 4).

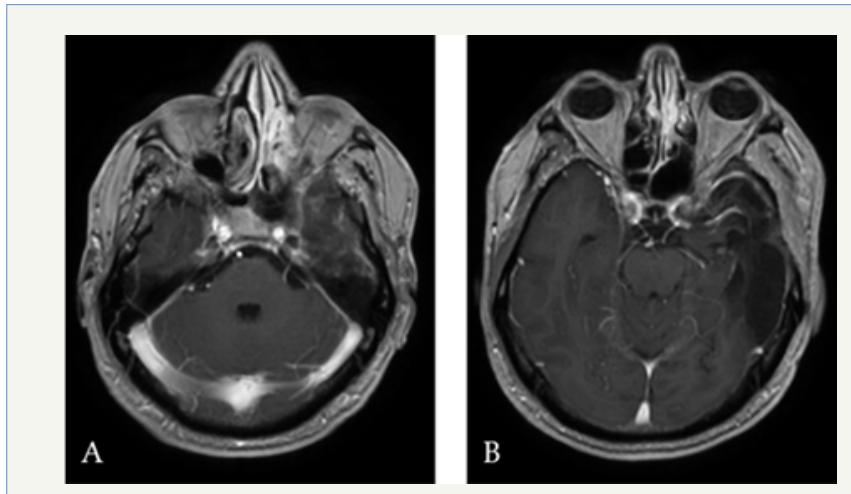

Figure 4: Contrast-enhanced axial T1-weighted MRI 22 months after surgery and medical treatment showing a clear regression of the tumor.

\section{Discussion}

Primary tumors derived from cells of glial line mainly affect the brain white matter. Glial tumors are usually confined to the intracranial compartment at the level of the cerebral parenchyma. The mechanism of these metastases remains imprecise, but glioblastoma metastases have been reported frequently at the 
pulmonary level, pleural, lymph node, bone and liver [3]. Several theories have been advanced: direct propagation via the dural vessels to the extra-meningeal tissues [4]; invasion through the dura mater, the bone, and cell migration via the ventricular system [5]; vascular invasion after radiotherapy [6] these include the dural linings which have a barrier function, the basal membranes surrounding the central nervous system vessels, and the direct destruction of the dura mater and cranial architecture [7]. Recent studies showing that the intracranial compartment contrary to what is believed is endowed with a lymphatic system [8], this may explain the extracranial development of glioblastoma metastases.

Some rare cases of glioblastomas destroying the surrounding bone by invasion of the extracellular matrix with extradural extension have been described. Thus Goodwin et al reported 28 published cases of spinal metastases of glioblastoma through the literature [2].

The propagation mechanism of glial tumors is not well known, the genetic examinations carried out in this field concerning the invasion of local glial tumors did not find genetic abnormalities.We don't have any explanation for such pattern of evolution, but this unusual GB behavior is very rare. Other plausible mechanisms have been put forward by Shenoy and Raja, who develop the hypothesis of a dural necrosis by disruption of blood supply and bone invasion [9].

The dura mater provides excellent protection against the infiltration of malignant tumors. It is extremely rare for glial tumor cells to infiltrate the dura mater and then invade adjacent structures. Recently, circulating tumor cells have been detected in the blood of $20-39 \%$ of patients with GB [10] which can explain a metastasis at a distance from the glioblastoma. More commonly, cranial metastasis by a glial tumor has been described in the presence of prior surgical intervention (craniotomy or biopsy) and/or irradiation [11]. It is much more common to observe this phenomenon with meningiomas, metastases or sarcomas that would cause direct extension to the skull with destruction of the dura mater.

When the dura mater is surgically disrupted, the extracerebral extension of malignant gliomas can be observed [12]. We believe that the opening of the dura mater facilitates the continuous exposure of extrameningeal tissue to glioma cells capable of invading the lymphatic vessels and extracerebral veins, strengthening the likelihood of widespread. The local invasion of the dura mater through the venous sinuses could play a role in the described cases.

The most frequent bone involvement in glioblastoma invasion is convexity and mean cerebral fossa. In our case described, the tumor is located in the temporal base and we believe it could penetrate the perivascular slit secondary to a chronic increased intracranial pressure.

For many years the prognosis of GB was considered poor with a median survival time of about 1 year. With standard treatment, median survival for adults with an anaplastic astrocytoma is about two to three years. For adults with the more aggressive glioblastoma, treated with concurrent temozolomide and radiation therapy, median survival is about 14.6 months with a two-year median survival rate of $27 \%$; five-year survival is $10 \%$. However, there are case reports of patients surviving for 10-20 years [13].

Our patient has undergone surgery and advanced chemotherapy regimens associated with radiotherapy. He obeys the criteria defining an extraneural glioblatoma multiform metastasis established by Weiss et al. [14]: the metastatic lesion must have the same histological characteristics of the cerebral neoplasm; The clinical evolution suggests that the first symptom were caused by the cerebral lesion; a complete absence of another primary tumor; The morphology of the tumor of the central nervous system (CNS) and the distant metastases must have been identical with the due allowance for difference in degrees of anaplasia.

Our patient is considered as long-term survivor because he has lived for 24 months after the first surgery. The individualized schedule of metronomic chemotherapy deserves particular considerations. This second line of chemotherapy used gave rise to a new perspective. The treatment has been well tolerated, and the correct response no longer allowed surgical approach in our patient.

\section{Conclusion}

Bone metastases of glioblastoma are rarely reported. The bone and dural involvement of the skull base in a neoplastic context must include the GB in the differential diagnosis. Imaging makes it possible to suspect GB in such cases, but the final diagnosis is established by immunohistochemical examination. Targeted therapy is of major interest in the management of GB metastasis to improve their prognosis.

\section{Disclosure Conflict of Interest}

None

\section{References}

1. Desjardins A (2015) Neuro-oncology: What is the optimal use of bevacizumab in glioblastoma? Nat Rev Neurol 11(8): 429-430.

2. Goodwin CR, Liang L, Abu-Bonsrah N, Hdeib A, Elder BD, et al. (2016) Extraneural Glioblastoma Multiforme Vertebral Metastasis. World Neurosurg 89: 578.e3-582.e3.

3. Hamilton JD, Rapp M, Schneiderhan T, Sabel M, Hayman A, et al. (2014) Glioblastoma multiforme metastasis outside the CNS: three case reports and possible mechanisms of escape. J Clin Oncol 1(32): 80-84.

4. Beauchesne P, Soler C, Mosnier JF (2000) Diffuse vertebral body metastasis from a glioblastoma multiforme: a technetium-99m Sestamibi single-photon emission computerized tomography study. J Neurosurg 93(5): 887-890.

5. Maccauro G, Spinelli MS, Mauro S, Perisano C, Graci C, Rosa M (2011) Physiopathology of spine metastasis. Int J Surg Oncol 2011: 107969.

6. Myers T, Egelhoff J, Myers M (1990) Glioblastoma multiforme presenting as osteoblastic metastatic disease: case report and review of the literature. AJNR Am J Neuroradiol 11(4): 802-803.

7. Kawano N, Yada K, Ogawa Y, Sasaki K (1977) Spontaneous transdural extension of malignant astrocytoma. Case report. Journal of Neurosurgery 47(5): 766-770. 
8. Louveau A, Smirnov I, Keyes TJ, Eccles JD, Rouhani SJ, et al. (2015) Structural and functional features of central nervous system lymphatic vessels. Nature 523(7560): 337-341.

9. Shenoy S, Raja A (2005) Spontaneous transdural spread of glioblastoma with atypical presentation. British Journal of Neurosurgery 19(1): 6165.

10. Lombard A, Goffart N, Rogister B (2015) Glioblastoma circulating cells: reality, trap or illusion? Stem Cells Int 2015: 182985.

11. Houston SC, Crocker IR, Brat DJ, Olson JJ (2000) Extraneural metastatic

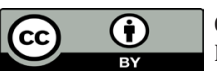

Creative Commons Attribution 4.0

International License

For possible submissions Click Here

Submit Article glioblastoma after interstitial brachytherapy. Int J Radiat Oncol Biol Phys 48(3): 831-836.

12. Horiuchi T, Osawa M, Itoh N, Kobayashi S, Nitta J, Hongo K (1996) Extradural extension of glioblastoma multiforme into the oral cavity: Case report. Surg Neurol 46(1): 42-46.

13. (2017) Glioblastoma and Malignant Astrocytoma. American brain tumor association p. 1-24.

14. Weiss L (1955) A metastasizing ependymoma of the cauda equina. Cancer 8: 161-171.

Your subsequent submission with Crimson Publishers will attain the below benefits

- High-level peer review and editorial services

- Freely accessible online immediately upon publication

- Authors retain the copyright to their work

- Licensing it under a Creative Commons license

- Visibility through different online platforms

- Global attainment for your research

- Article availability in different formats (Pdf, E-pub, Full Text)

- Endless customer service

- Reasonable Membership services

- Reprints availability upon request

- One step article tracking system 